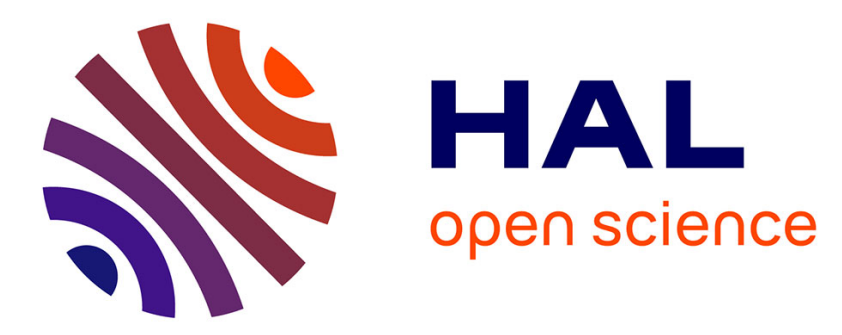

\title{
Fluctuations and stratification in sedimentation of dilute suspensions of spheres
}

Daniel Chehata Gomez, Laurence Bergougnoux, Elisabeth Guazzelli, E. John Hinch

\section{- To cite this version:}

Daniel Chehata Gomez, Laurence Bergougnoux, Elisabeth Guazzelli, E. John Hinch. Fluctuations and stratification in sedimentation of dilute suspensions of spheres. Physics of Fluids, 2009, pp.093304. 10.1063/1.3231834 . hal-01066996

\section{HAL Id: hal-01066996 https://hal.science/hal-01066996}

Submitted on 22 Sep 2014

HAL is a multi-disciplinary open access archive for the deposit and dissemination of scientific research documents, whether they are published or not. The documents may come from teaching and research institutions in France or abroad, or from public or private research centers.
L'archive ouverte pluridisciplinaire HAL, est destinée au dépôt et à la diffusion de documents scientifiques de niveau recherche, publiés ou non, émanant des établissements d'enseignement et de recherche français ou étrangers, des laboratoires publics ou privés. 


\title{
Fluctuations and stratification in sedimentation of dilute suspensions of spheres
}

\author{
Daniel Chehata Gómez, ${ }^{1}$ Laurence Bergougnoux, ${ }^{1}$ Élisabeth Guazzelli, ${ }^{1}$ and John Hinch ${ }^{2}$ \\ ${ }^{1} I U S T I$ CNRS UMR 6595, Polytech'Marseille, Aix-Marseille Université (U1), 5 Rue Enrico Fermi, \\ 13453 Marseille Cedex 13, France \\ ${ }^{2}$ DAMTP, University of Cambridge, Wilberforce Road, Cambridge CB3 OWA, United Kingdom
}

(Received 8 June 2009; accepted 3 August 2009; published online 24 September 2009)

\begin{abstract}
We have tested in experiments and simulations whether stratification can control velocity fluctuations in suspensions of sedimenting spheres. The initial value and early decay of the velocity fluctuations are not affected by stratification. On the other hand, in the descending front where the stratification is strong and well defined, the velocity fluctuations are inhibited according to a previously proposed scaling. In between, after the initial decay and before the arrival of the front, the local value of the stratification does not always play a role. (C) 2009 American Institute of Physics. [doi:10.1063/1.3231834]
\end{abstract}

\section{INTRODUCTION}

In quiescent sedimentation under Stokes conditions, the settling speed of a particle is not solely characterized by a mean vertical velocity but needs to be supplemented by a fluctuation due to the constantly changing configuration of the suspension microstructure. The mean velocity is found to be proportional to the Stokes velocity for a single isolated sphere $V_{S}=2 a^{2}\left(\rho_{p}-\rho_{f}\right) g / 9 \mu$, where $a$ is the sphere radius, $\rho_{p}$ is the density of the particles, $\rho_{f}$ is that of the fluid, and $\mu$ is the fluid viscosity. The proportionality factor $f(\phi)$ is seen experimentally to be a decreasing function of the volume fraction $\phi .{ }^{1}$ On the theoretical side, the long range nature of the hydrodynamic interactions between the particles can lead to divergent integrals when summing the contributions induced on a test sphere from all the other spheres. The problem of the divergent integrals for the mean velocity was solved by Batchelor ${ }^{2}$ who found the first correction $f(\phi)=1-6.55 \phi+O\left(\phi^{2}\right)$ in concentration to the Stokes velocity assuming low $\phi$ and randomly dispersed spheres. However, this divergence problem remains for the velocity fluctuations. Extending the analysis of Batchelor for a dilute random distribution of sedimenting spheres, Caflisch and Luke $^{3}$ found that the variance in settling speed diverges with the size of the container. ${ }^{4}$ While being very large and anisotropic, ${ }^{5,6}$ the velocity fluctuations measured in experiments are not seen to diverge with the size of the container. ${ }^{5,7,8}$ In large containers, ${ }^{9,10}$ large-scale fluctuations of the size of the width of the cell indeed dominate the dynamics just after the initial mixing of the suspension. However they are transient and smaller-scale fluctuations of size $\approx 20 a \phi^{-1 / 3}$ remain and prevail until the arrival of the upper sedimentation front. ${ }^{8-10}$ This divergence paradox has prompted several theoretical studies, some suggesting that a vertical concentration stratification may inhibit the diverging fluctuations. ${ }^{11-15}$

Some understanding of the fluctuation divergence can be found by a scaling argument developed by Hinch. ${ }^{4}$ Blobs of length $l$, varying from the cross-sectional size of the con- tainer $L$ down to the interparticle spacing $a \phi^{-1 / 3}$, contain on average $N_{l}=\phi l^{3} / a^{3}$ particles. Random mixing produces fluctuations in the weight of the blob of $\sqrt{N_{l}} F$, where $F=\frac{4}{3} \pi a^{3}\left(\rho_{p}-\rho\right) g$ is the excess weight of one sphere. Balancing this fluctuation in the weight against the Stokes drag on the blob yields an estimate of the convection currents, also called "swirls," of

$$
V^{\prime}(l)=\frac{\sqrt{N_{l}} F}{6 \pi \mu l}=V_{S} \sqrt{\phi l / a} .
$$

Hence just after mixing, the dominant velocity fluctuations come from the largest possible blobs, those on the size of the container, $l=L$. All the containers in our experiments and simulations have a square cross section $L \times L$ and a height $H$ larger than $L$. For containers with a depth $D$ smaller than the width $W$ that is smaller than the height $H$, the dominant velocity fluctuations come from blobs the size of the depth, $l=D$. With $l=L$ the velocity fluctuations have the Poisson velocity scaling

$$
V_{\text {Poisson }}=V_{S} \sqrt{\phi L / a} .
$$

It is interesting to note that this velocity depends only on the force $F$, the viscosity $\mu$, the size of the container $L$, and the number density $3 \phi / 4 \pi a^{3}$, and does not depend on the size of the particles $a$.

The large-scale fluctuations dominate the dynamics initially but they are transient ${ }^{9}$ as heavy blobs settle to the bottom and light blobs rise to the top. The relaxation time of these large-scale fluctuations is then given that the time a blob falls the size of the container $L$,

$$
\tau_{\text {relax }}=\frac{L}{V_{\text {Poisson }}}=t_{S} \sqrt{L / a \phi},
$$

where $t_{S}=a / V_{S}$ is the time an isolated sphere takes to settle through its radius. Hinch ${ }^{4}$ suggested that the strong initial convection current might remove fluctuations in the number density down to the irreducible scale of interparticle separations $a \phi^{-1 / 3}$. 
TABLE I. Particle characteristics: mean radius $\langle a\rangle$ and standard deviation $\sigma_{a}$, sphere density $\rho_{p}$, Stokes velocity $V_{S}$, and sphere Reynolds number $\operatorname{Re}_{p}=V_{S}\langle a\rangle \rho_{f} / \mu$.

\begin{tabular}{lccccc}
\hline \hline Batch & $\begin{array}{c}\langle a\rangle \\
(\mathrm{cm})\end{array}$ & $\begin{array}{c}\frac{\sigma_{a}}{\langle a\rangle} \\
(\%)\end{array}$ & $\begin{array}{c}\rho_{p} \\
\left(\mathrm{~g} / \mathrm{cm}^{3}\right)\end{array}$ & $\begin{array}{c}V_{S} \\
(\mathrm{~cm} / \mathrm{s})\end{array}$ & $\begin{array}{c}\mathrm{Re}_{p} \\
\left(\times 10^{-5}\right)\end{array}$ \\
\hline $\mathrm{A}(\bigcirc)$ & 0.0202 & 2.5 & $2.50 \pm 0.04$ & $0.0136 \pm 0.0009$ & 2.7 \\
$\mathrm{~B}(\square)$ & 0.0152 & 2.5 & $4.11 \pm 0.07$ & $0.0158 \pm 0.0010$ & 2.3 \\
$\mathrm{C}(\triangle)$ & 0.0148 & 5.6 & $4.11 \pm 0.07$ & $0.0150 \pm 0.0018$ & 2.1 \\
$\mathrm{D}(\diamond)$ & 0.0187 & 11.3 & $2.50 \pm 0.04$ & $0.0117 \pm 0.0027$ & 2.1 \\
\hline \hline
\end{tabular}

A scaling argument ${ }^{11,13-16}$ also shows how stratification may suppress the velocity fluctuations. When there is a vertical variation in the concentration $d \phi / d z$, a blob of size $l$ with excess weight $\sqrt{N_{l}} F$ will fall a distance $\delta z$ to find its level of neutral buoyancy, with $\delta z$ given by

$$
-\delta z \frac{d \phi}{d z} \frac{N_{l}}{\phi} F=\sqrt{N_{l}} F .
$$

This distance becomes smaller than the size of the blob for blobs larger than a stratification cutoff size

$$
l_{s}=a \phi^{1 / 5}\left(-a \frac{d \phi}{d z}\right)^{-2 / 5}=a \phi^{-1 / 5}(a \beta)^{-2 / 5},
$$

introducing the stratification parameter $a \beta=-(a / \phi) d \phi / d z$. The largest remaining velocity fluctuations are then on the following cutoff scale:

$$
V^{\prime}\left(l_{s}\right)=V_{S} \phi^{3 / 5}\left(-a \frac{d \phi}{d z}\right)^{-1 / 5}=V_{S} \phi^{2 / 5}(a \beta)^{-1 / 5} .
$$

It is interesting to note that this velocity also depends on the force $F$, the viscosity $\mu$, the number density $3 \phi / 4 \pi a^{3}$, and the stratification $\beta$, and does not depend on the size of the particles $a$.

The above argument for control of fluctuations by stratification applies only when the cutoff length is smaller than the cross-sectional size of the container, i.e., for $l_{s}<L$. There is thus critical stratification $\beta_{c}$ for $l_{s}=L$,

$$
a \beta_{c}=\left(\frac{a}{L}\right)^{5 / 2} \phi^{-1 / 2} \text {. }
$$

When stratification is larger than this critical value, stratification should always control the fluctuations. Following Mucha et al., ${ }^{15}$ we can express the scaling for the fluctuations in terms of $\beta_{c} / \beta$,

$$
V^{\prime}\left(l_{s}\right)=V_{\text {Poisson }}\left(\beta_{c} / \beta\right)^{1 / 5},
$$

and the cutoff length can be written as

$$
l_{s}=L\left(\beta_{c} / \beta\right)^{2 / 5} .
$$

Coming back to the relaxation time of the large-scale fluctuations, instead of a blob falling the size of the container $L$, one might expect the blob to fall just to its neutrally buoyant level, i.e., the relaxation time becomes

$$
\frac{l_{s}}{V^{\prime}\left(l_{s}\right)}=t_{S} \sqrt{\frac{L}{a \phi}}\left(\frac{\beta_{c}}{\beta}\right)^{2 / 5} .
$$

The objective of this paper is to examine both in experiments and simulations whether or not the reduction in the velocity fluctuations is controlled by stratification. A preliminary account of some of the experiments has already been published. ${ }^{17}$ Here, we present a detailed account of our results for both the velocity fluctuations and the concentration gradient, including effects of polydispersity, which may be crucial in interpreting experimental results. It is indeed found that a reproducible mixing procedure interacts with particles of different polydispersities to produce different initial gradients. The influence of this initial gradient and of its further evolution on the velocity fluctuations is examined and the proposed stratification scaling tested. In the simulations, it is easier to produce a given and controlled linear stratification and examine its influence on the velocity fluctuations. It is also possible to produce a uniform initial suspension and inspect the velocity fluctuations as the sedimentation front passes the measuring window as in the experiments.

\section{EXPERIMENTAL TECHNIQUES}

Four different batches of glass spheres, supplied by Cataphote Inc. and Potters Industries, were used to perform the experiments, see their characteristics in Table I. The fluid used was silicone oil $47 \mathrm{~V} 1000$ supplied by Rhodia which had a viscosity $\mu=10.0 \pm 0.3 \mathrm{P}$ and a density $\rho_{f}=0.965 \pm 0.007 \mathrm{~g} \mathrm{~cm}^{-3}$ at the air-conditioned room temperature of $25 \pm 1{ }^{\circ} \mathrm{C}$.

Most of the experiments were performed at the same initial volume fraction $\phi_{0}=0.3 \%$, in glass-wall vessels of different cross sections $\left(20 \times 20,10 \times 10\right.$, and $4 \times 4 \mathrm{~cm}^{2}$ for particles of batch $\mathrm{C}$ and $10 \times 10 \mathrm{~cm}^{2}$ for particles of batches $\mathrm{A}, \mathrm{B}$, and D) but filled with the same fluid height of $H=40 \mathrm{~cm}$. We also performed an additional series of experiments with particles of batch $\mathrm{B}$ in the cell having a cross section of $10 \times 10 \mathrm{~cm}^{2}$ for volume fractions varying from $0.1 \%$ to $0.8 \%$ by steps of $0.1 \%$. Particle velocity fluctuations as well as average concentration and vertical concentration gradient were measured during the sedimentation process in the same imaging window covering approximately a quarter of the height $H$.

In a first set of experiments, particle velocities were measured using particle image velocimetry. A thin light sheet 

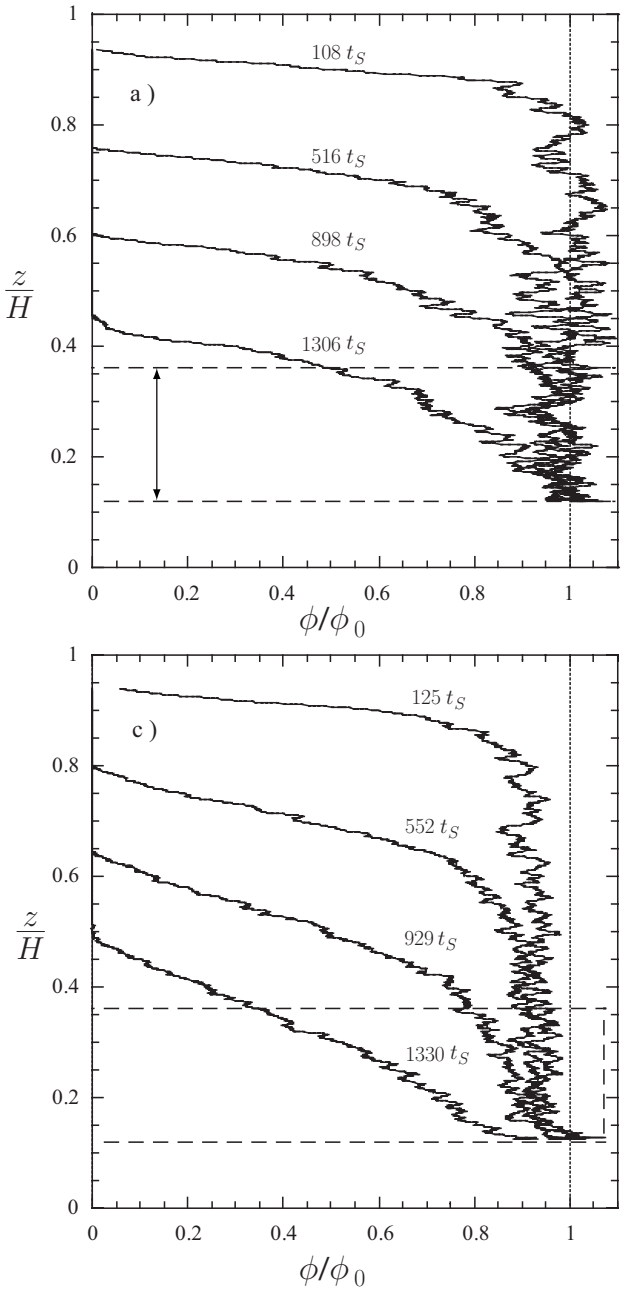
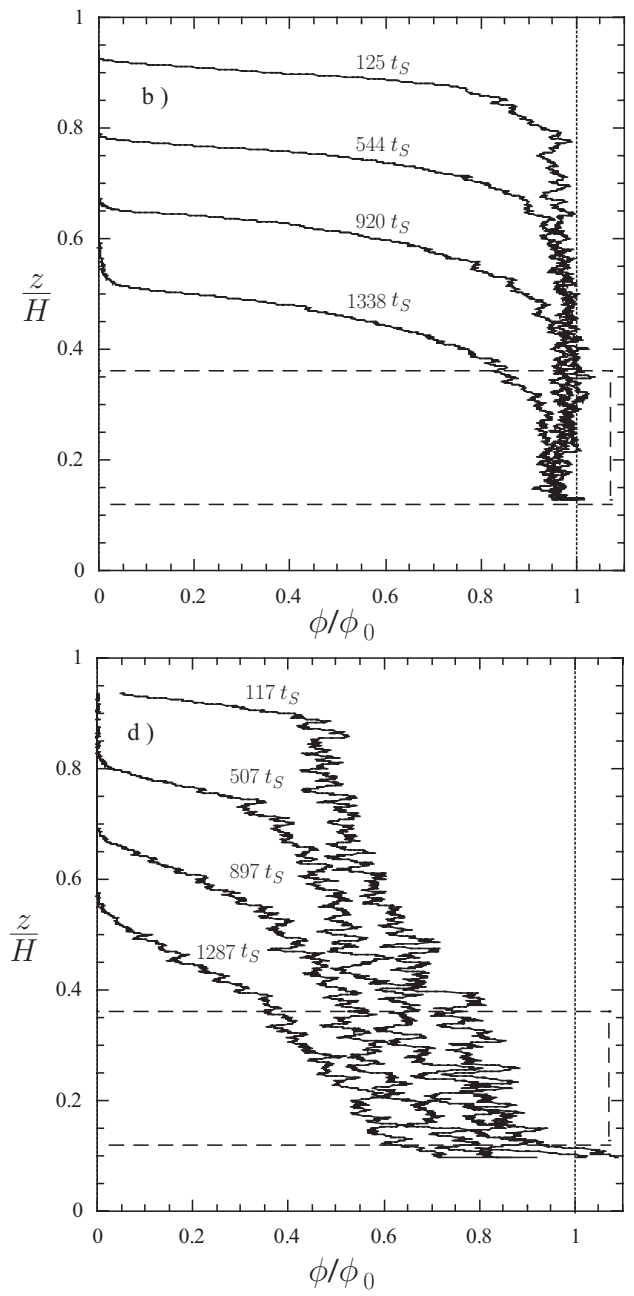

FIG. 1. Concentration profile vs normalized height $z / H$ for particles of batches A (a), B (b), D (c) in the cell having a cross section $10 \times 10 \mathrm{~cm}^{2}$, and C (d) in the smallest cell having a cross section of $4 \times 4 \mathrm{~cm}^{2}$ with $\phi_{0}=0.3 \%$. Each measurement of concentration was made on a volume equal to the horizontal section and a height of $0.3 \mathrm{~mm}$.

(of thickness $\approx 1 \mathrm{~mm}$ ) produced by two $15 \mathrm{~mW}$ laser diodes facing each other was employed to illuminate the median plane of the cell. A $(1040 \times 1392$ pixels $)$ charge coupled device digital camera (Basler A102f) placed at right angles to the light sheet was focused on the illuminated particles and sampled the entire cross section of the cell for a window of height $10 \mathrm{~cm}$ placed $25.5 \mathrm{~cm}$ below the liquid-air interface. The spatial resolution of the measurement was $\approx 0.7 \times 0.7 \mathrm{~cm}^{2}$ in the cell of cross sections $20 \times 20$ and $\approx 0.5 \times 0.5 \mathrm{~cm}^{2}$ in the smallest cells. During sedimentation, pairs of images separated by $\approx a / V_{S}$ were captured every 40 $\mathrm{s}$ and were processed to obtain a two-dimensional velocityvector map as described previously by Bergougnoux et al. ${ }^{10}$ and Chehata Gómez et al. ${ }^{17}$ At each captured time, we computed the vertical mean velocity and standard deviation $w^{\prime}$ from all velocity data coming typically from ten experimental runs.

In a second set of experiments, the particle concentration was obtained by means of light attenuation measurement through the suspension within the same imaging window but with the cell backlit by two neon tubes, as described by Chehata Gómez et al. ${ }^{17,18}$ Averaging the gray-scale intensities over the entire imaging window gives the average con- centration $\phi$. Summing the intensities over the horizontal lines of the image yields the vertical intensity profile. A linear fit of this profile gives the vertical gradient $-d \phi / d z$. Statistical analysis over typically three experimental runs provided the mean value and standard deviation of $\phi$ and $-d \phi / d z$.

Each sedimentation experiment consisted of mixing the suspension and then performing one of the measurements described above during the sedimentation process (the starting time corresponding to the cessation of mixing). It is difficult to make a reproducibly mixed homogeneous suspension. The mixing was thus always performed in the same way by moving a small propeller (of size $\approx 2 \mathrm{~cm}$ and speed $2000 \mathrm{rpm}$ ) within the suspension for $\approx 10 \mathrm{~min}$. First, the sediment was turned into a concentrated homogeneous suspension in the bottom $5 \mathrm{~cm}$ of the vessel. Second, the mixing was extended to a volume twice as high. Then, this procedure of doubling the mixed volume was repeated until the complete volume of the cell was found to be visually homogeneous.

However, this same mixing procedure produces reproducibly different gradients for the different batches of particles. For the polydisperse particles (batches $\mathrm{C}$ and $\mathrm{D}$ ), the 

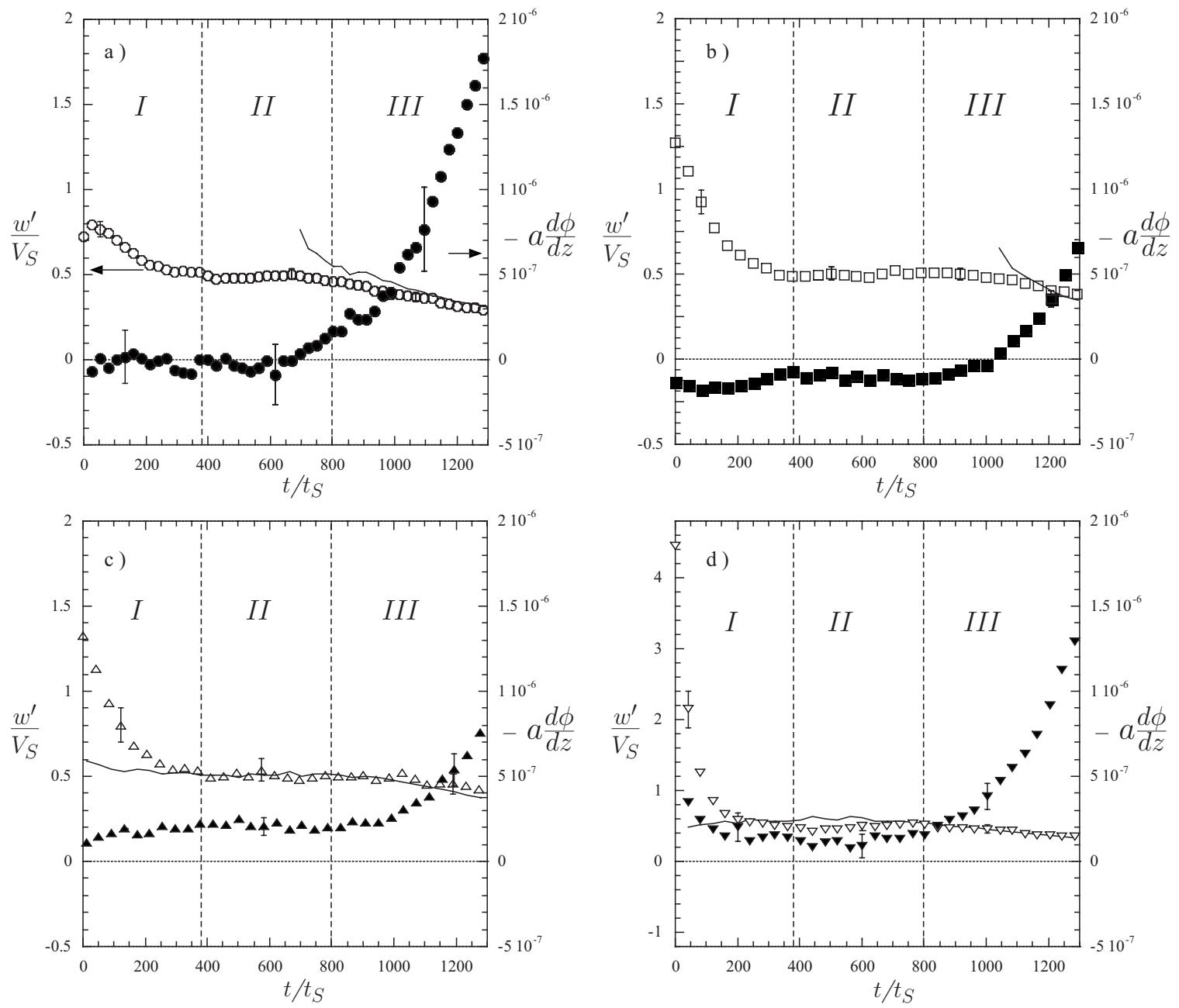

FIG. 2. Time evolution of the velocity fluctuations $w^{\prime}$ (open symbols) and the concentration gradient $-a d \phi / d z$ (filled symbols) for particles of batches A (a), B (b), C (c) in the cell having a cross section $10 \times 10 \mathrm{~cm}^{2}$, and C (d) in the cell having a cross section of $20 \times 20 \mathrm{~cm}^{2}$ with $\phi_{0}=0.3 \%$.

initial vertical gradient $-d \phi / d z$ is found to be always positive (stably stratified) while for the most monodisperse particles (batches A and B), it fluctuates about zero and can even be negative (unstably stratified) in the imaging window and slightly positive above (for batch B for the different $\phi_{0}$ studied), see Figs. 1(a)-1(c). It seems as if a fixed preparation method interacts with particles of different polydispersities to produce different initial gradients, more precisely the gradient becomes positive for more polydisperse particles. In the smallest cell having a cross section of $4 \times 4 \mathrm{~cm}^{2}$ in which the propagation of the mixing along the cell happens not to be completely effective, the initial gradient is large but reproducible, see Fig. 1(d).

\section{EXPERIMENTAL RESULTS}

Figure 2 shows the time evolution of the velocity fluctuations and concentration gradient for particles of different polydispersities (batches A-C) in the same cell of cross section $10 \times 10 \mathrm{~cm}^{2}$ and for particles of batch $\mathrm{C}$ in two different cells of cross sections $10 \times 10$ and $20 \times 20 \mathrm{~cm}^{2}$ (the time has been normalized by the Stokes time $t_{S}=a / V_{S}$ and the velocities by the Stokes velocity $V_{S}$ ). The plots are terminated at $t=1300 t_{S}$, at which time the sedimentation front in the imaging window is no longer well represented by a linear variation of the concentration.

The velocity fluctuations present a very robust evolution with an early strong decrease (region I) followed by a plateau (region II) whatever initial gradient is created by the mixing of particles having different polydispersities (gradient fluctuating about zero for particles of batch A, negative gradient for those of batch $\mathrm{B}$, and positive for those of batch C). The plateau region is followed by a small decrease (region III) for $t / t_{S} \geq 900$ owing to a strong increase in the gradient when the sedimentation front reaches the imaging window. The stratification prediction for the fluctuations $\propto \phi^{3 / 5}(-a d \phi / d z)^{-1 / 5}$ (solid line in Fig. 2 with the adjustable numerical factor being kept at a value of 0.8 ) has only been computed for positive values of the vertical gradient $-a d \phi / d z$ as the scaling contains a noninteger power law. A reasonable agreement is achieved in the front.

Stratification has clearly no effect on the initial value and early decrease in the velocity fluctuations and interestingly has also no effect on the long time plateau value because it is identical $\approx 0.5 V_{S}$ for different polydispersities and cells. In this regard, it is particularly interesting to compare Figs. 2(b) 

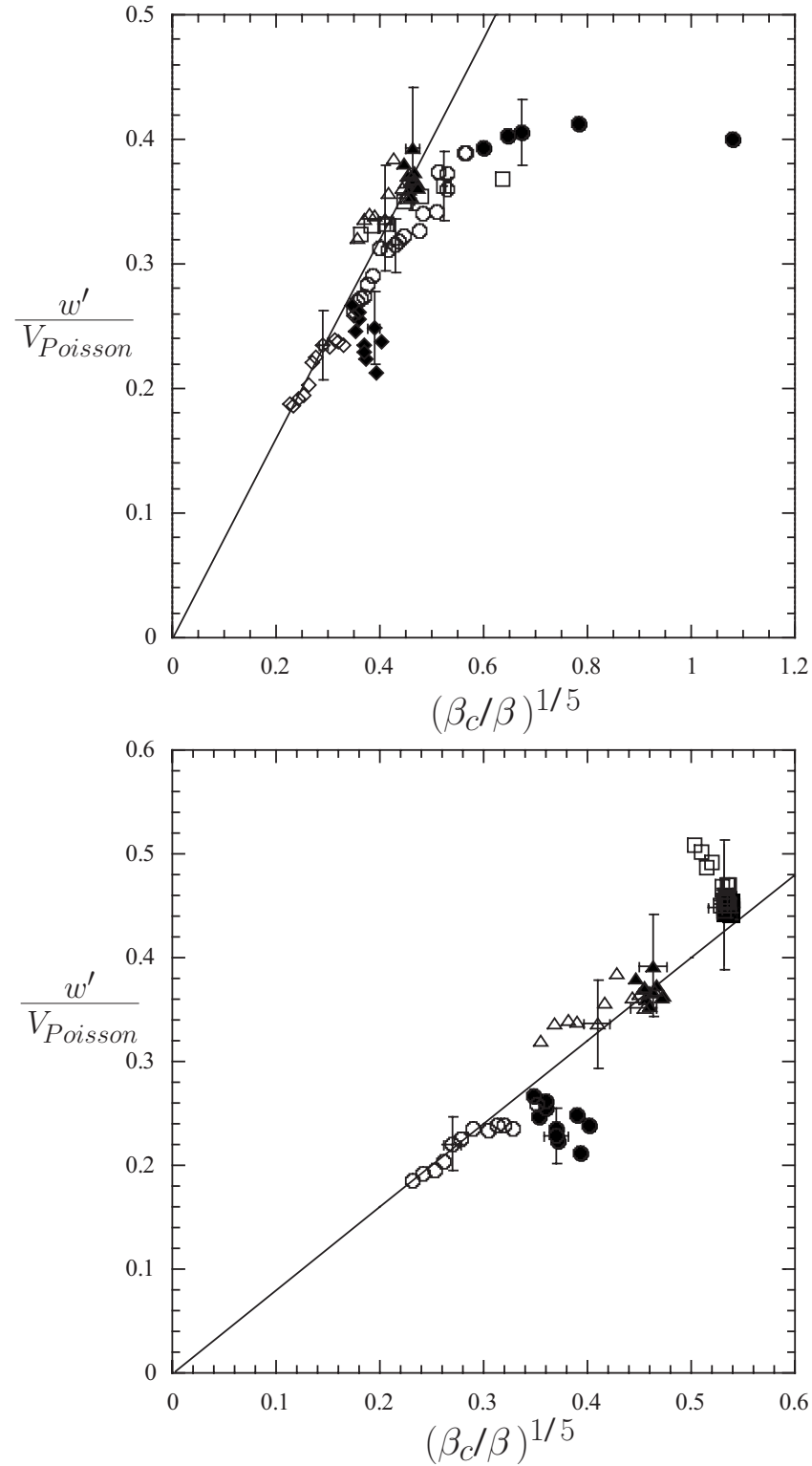

FIG. 3. Plateau (filled symbols) and front (open symbols) velocity fluctuations $w^{\prime}$ normalized by Poisson scaling $V_{\text {Poisson }}$ vs $\left(\beta_{c} / \beta\right)^{1 / 5}$; (top) for particles of batches $\mathrm{A}(\bigcirc), \mathrm{B}(\square), \mathrm{C}(\triangle)$, and $\mathrm{D}(\diamond)$ in the same cell having a cross section $10 \times 10 \mathrm{~cm}^{2}$ with $\phi_{0}=0.3 \%$; and (bottom) for particles of batch $\mathrm{C}$ in the different cells having cross sections $4 \times 4 \mathrm{~cm}^{2}(\square)$, $10 \times 10 \mathrm{~cm}^{2}(\triangle)$, and $20 \times 20 \mathrm{~cm}^{2}(\bigcirc)$ with $\phi_{0}=0.3 \%$.

and 2(c) for which particles made of the same material but having different polydispersities (particles of batch B are sieved more carefully than those of batch $\mathrm{C}$ ) settle in the same cell of cross section $10 \times 10 \mathrm{~cm}^{2}$ with same $\phi_{0}=0.3 \%$. As previously noted by Chehata Gómez et al. ${ }^{17}$ the velocity fluctuations are identical despite the difference in initial stratification created by mixing (negative and positive gradients for batches B and C, respectively). It is also interesting to compare Figs. 2(c) and 2(d) for which particles of the same batch $\mathrm{C}$ settle in different cells with same $\phi_{0}=0.3 \%$. The initial value and early decrease in the velocity fluctuations greatly differ despite same initial stratification.

Figure 3 (top) shows, for particles of different polydispersities in the same cell and same $\phi_{0}=0.3 \%$, the plateau and front values of the velocity fluctuations normalized by the

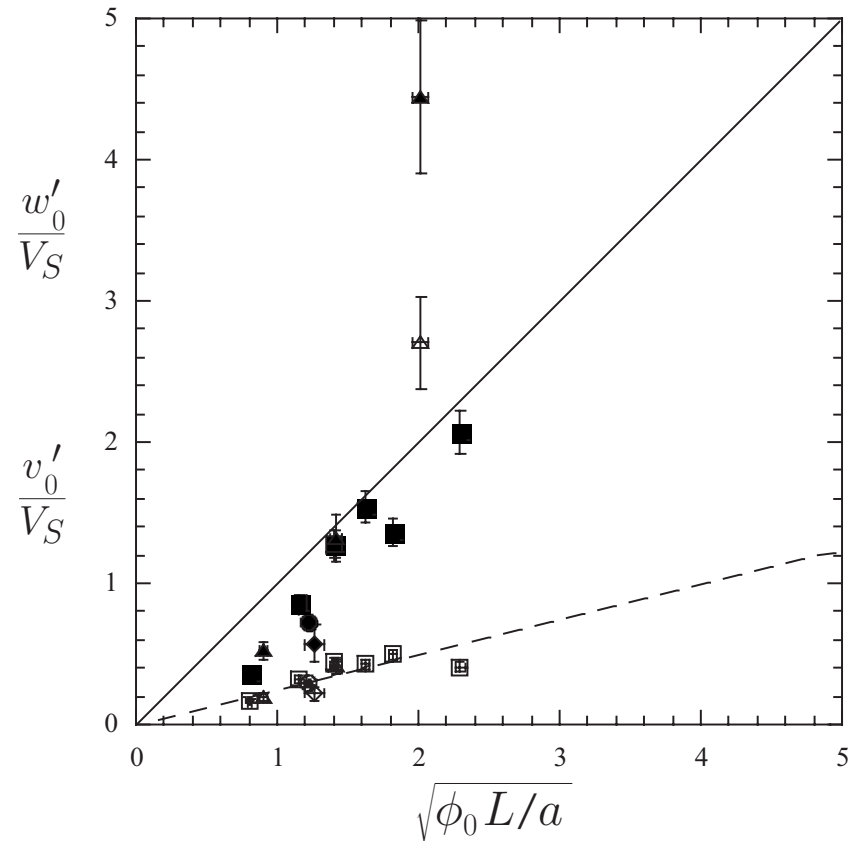

FIG. 4. Initial velocity fluctuations $w_{0}^{\prime}$ (filled symbols) and $v_{0}^{\prime}$ (open symbols) vs Poisson value $V_{S} \sqrt{\phi_{0} L / a}$ for the particles of the different batches A $(\bigcirc)$, B ( $\square$ ) for different $\phi_{0}, \mathrm{C}(\triangle)$ for different cells, and D $(\diamond)$. The solid line corresponds to a slope of value 1 while the dashed line to a slope of value $\frac{1}{4}$.

Poisson scaling $V_{\text {Poisson }}=V_{S} \sqrt{\phi L / a}$ as a function of the stratification $a \beta=-(a / \phi) d \phi / d z$ and the critical stratification $a \beta_{c}=(a / L)^{5 / 2} \phi^{-1 / 2}$ in the proposed stratification scaling (4). While the data in the front collapse approximately onto the same line,

$$
w^{\prime}=0.8 V_{\text {Poisson }}\left(\beta_{c} / \beta\right)^{1 / 5},
$$

the plateau data do not seem to satisfy the proposed scaling. This is particularly evident for particles of batches A and D. Note that this scaling analysis can only be undertaken for positive values of the stratification and thus there is no data from the plateau for batch B. Note also that the stratification is always much larger than critical stratification for the plotted data. Figure 3 (bottom) presents the same test of the stratification scaling for the same particles of batch $\mathrm{C}$ with $\phi_{0}=0.3 \%$ in cells of different cross sections. A collapse onto the same line is approximately obtained for the front data but not for the plateau data (in particular in the largest cell) even though stratification is larger than critical stratification by a factor $\gtrsim 10^{2}$ in this region.

Now that we have discussed the velocity fluctuations in the plateau and the front in terms of the proposed scaling, we turn to the initial value and early decay of the fluctuations (we include in this analysis the data from batch B at different $\phi_{0}$ which were not used above because of their negative initial gradients). The initial vertical velocity fluctuations $w_{0}^{\prime}$ clearly increase with increasing $L / a$ and $\phi_{0}$. They approximately satisfy the Poisson scaling of an homogeneous suspension (1) with a coefficient of unity,

$$
w_{0}^{\prime} \approx 1.0 V_{\text {Poisson }} \text {, }
$$

as seen in Fig. 4. The same trend is followed by the initial 

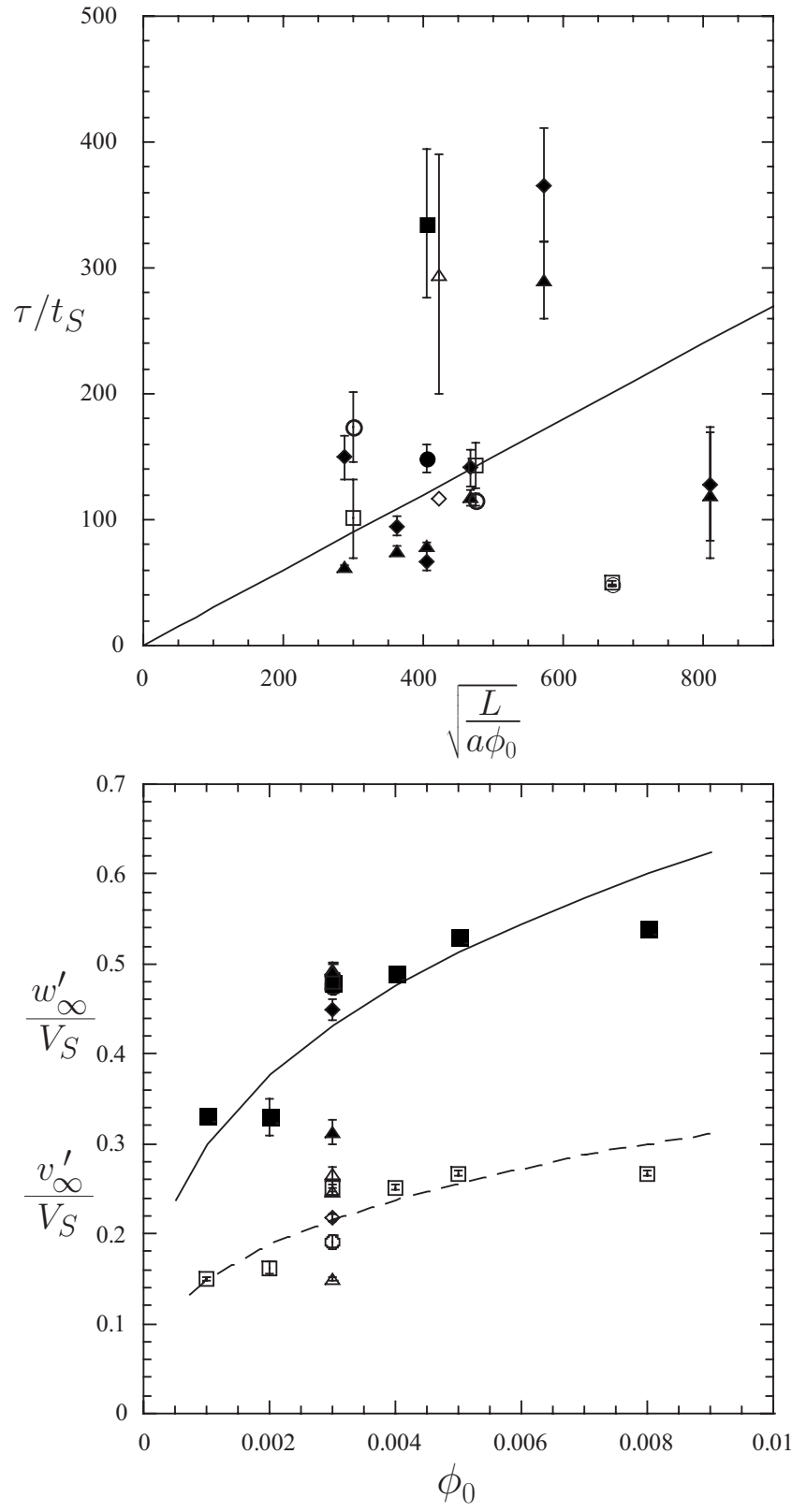

FIG. 5. Normalized decay time $\tau / t_{S}$ vs $\sqrt{L / a \phi_{0}}$ (top) and plateau fluctuations vs $\phi_{0}$ (bottom) for the particles of the different batches A (O), B ( $\square$ ) for different $\phi_{0}, \mathrm{C}(\triangle)$ for different cells, and D $(\diamond)$. The filled symbols correspond to the vertical direction and the open symbols to the horizontal direction. The solid line corresponds to the curve $3 \phi^{1 / 3}$ while the dashed line to $1.5 \phi^{1 / 3}$.

horizontal velocity fluctuations $v_{0}^{\prime}$. However the magnitude of the initial fluctuations is four times larger in the vertical direction than in the horizontal direction.

In order to find the decay time $\tau$ of the initial fluctuations, the strong early decrease in the fluctuations is fitted to a simple exponential curve $w_{\infty}^{\prime}\left(\right.$ or $\left.v_{\infty}^{\prime}\right)+C \exp (-t / \tau)$ between $0 \leq t / t_{S} \leq 800$. The correlation coefficients of the fits are larger than 0.99 except for the case of batch D. The decay time seems independent of the initial stratification, as previously noted in comparing Figs. 2(b) and 2(c) where the same decay is found whatever (negative or positive) initial gradient is created by the mixing. Figure 5 (top) plots $\tau / t_{S}$ versus $\sqrt{L / a \phi_{0}}$ corresponding to a blob of size of the container $L$ falling its size with its initial Poisson fluctuations $V_{S} \sqrt{\phi_{0} L / a}$, see Eq. (2) in Sec. I. Although the data are very scattered, there might be a linear variation

$$
\tau \approx 0.3 t_{S} \sqrt{L / a \phi} .
$$

The plateau values $w_{\infty}^{\prime}$ and $v_{\infty}^{\prime}$ of the velocity fluctuations have been found to be independent of polydispersity and cell size, as well as the initial stratification in the preceding discussion of Figs. 2 and 3. The plateau values $w_{\infty}^{\prime}$ increase slightly with increasing volume fraction, roughly as the $\phi^{1 / 3}$ power law found by Segrè $e t a l .{ }^{8}$ However, the coefficient of the power law differs as $w_{\infty}^{\prime} \approx 3 V_{S} \phi^{1 / 3}$ in the present case and $w_{\infty}^{\prime} \approx 2 V_{S} \phi^{1 / 3}$ in the experiments of Segrè et al. ${ }^{8}$ in thin cells. Note that the anisotropy of the fluctuations in the plateau is $w_{\infty}^{\prime} / v_{\infty}^{\prime} \approx 2$, as previously seen by Nicolai et al. ${ }^{6}$ and Segrè et $a .^{8}$

\section{COMPUTER SIMULATIONS}

Two series of numerical experiments were performed. In the first, the initial suspension was linearly stratified and the velocity fluctuations were monitored in a central window well away from the descending front between the suspension and the clear fluid above. In the second, the initial suspension was uniform and the velocity fluctuations were monitored as the front passed through a vertical series of windows.

The computer simulations were made with the method of Bergougnoux et al. ${ }^{10}$ also used by Chehata Gomez et al. ${ }^{18}$ Here only monodisperse simulations were made. The hydrodynamic interactions are calculated treating the particles as point particles which exert a force equal to their weight compensated for buoyancy. Point particles can be used because as we observed in the introduction, the velocity fluctuations of interests (1) and (4) do not depend on the size of the particles.

The induced Stokes flow is represented by a finite sum of Fourier modes. Each Fourier mode satisfies boundary conditions of no flow normal through the boundaries and no tangential stress. The spatial distribution of the point forces exerted by the distributed particles is thus broken down into appropriate Fourier modes of force which then drive the Fourier modes of the flow. This flow is evaluated at each particle in order to move the particles.

The simulations were conducted in a box of height $H$, width $L$, and depth $L$. The number of Fourier modes used was $n_{F}$ in each horizontal direction and $n_{F} H / L$ in the vertical direction. In many of the simulations, the number of particles $N$ was set equal to the total number of Fourier modes, $n_{F}^{3} H / L$. This means that the flow was well resolved for length scales from the size of the box, $L$, down to the interparticle separation, $\left(L^{2} H / N\right)^{1 / 3}$. Smaller length scales, down to the particle diameter, were not resolved at all.

As a result of the limited spatial resolution, a single particle does not sediment at its correct Stokes settling velocity, $F / 6 \pi \mu a$, but instead on average at $0.15 F n_{F} / \mu L$, where $F$ is the force on the particle, $\mu$ is the viscosity of the fluid, and $a$ is the radius of the spherical particle. In order to have the particles sediment at their correct velocity, it is therefore nec- 
essary to add a correction (not included by Mucha et al. ${ }^{15}$ ) of $F / 6 \pi \mu a-0.15 F n_{F} / \mu L$ to the hydrodynamically induced flow from the limited number of Fourier modes. This correction introduces the parameter $a$, the "radius" of the particle. Although the radius exists in the expression above, it is elsewhere ignored so that the hydrodynamic interactions are calculated for point particles, and also no effects of excluded volume are included.

The numerical simulations are nondimensionalized by scaling lengths on $L$, velocities on $F / \mu L$, and so time on $\mu L^{2} / F$. The nondimensional sedimentation velocity then becomes

$$
V=\frac{L}{6 \pi a} .
$$

While this is clearly the ratio of the size of the box to the size or the particles, we are ignoring the size of the particles except in this sedimentation velocity. In the simulations in this paper, we set $V=2$. We set the nondimensional height $h=H / L$. An important parameter is the nondimensional number density of particles $n=N / h$, which is related to dimensional quantities by $n=3 \phi L^{3} / 4 \pi a^{3}$. It should be noted that the number of particles in one simulation is typically $10^{4}$ compared to $10^{6}$ in one laboratory experiment. Averages are however taken typically over 320 different realizations, more than the ten of the experiments.

\section{NUMERICAL RESULTS IN INITIALLY STRATIFIED SUSPENSION}

In the first series of numerical experiments, we study the evolution of the velocity fluctuations in a central window starting with a stratified suspension. The particles were initially positioned uniformly randomly in the horizontal plane, and with a bias in the vertical direction so as to achieve a linear stratification. In more detail, $\zeta$ was chosen uniformly randomly on $[0,1]$, and the vertical position of the particle set as

$$
z=h \frac{2 \zeta}{1+\frac{1}{2} b h+\sqrt{\left(1+\frac{1}{2} b h\right)^{2}-2 b h \zeta}} .
$$

This gives linear variation of the concentration of the particles

$$
\phi=\phi_{0}\left[1-b\left(z-\frac{1}{2} h\right)\right] .
$$

The nondimensional stratification is therefore

$$
b=-\frac{1}{\phi_{0}} \frac{\partial \phi}{\partial z},
$$

and this is related to dimensional quantities by $b=L \beta$.

The height of the simulation box was set at $h=10$ for the first series of numerical simulations. The velocity fluctuations were monitored in a window $2<z<6$ during a time $0<t<0.5$. By $t=0.5$, the center of the front drops to $z=9$ and the width of the front grows to typically 1 . Hence no effect of the front on the concentration can be seen within the

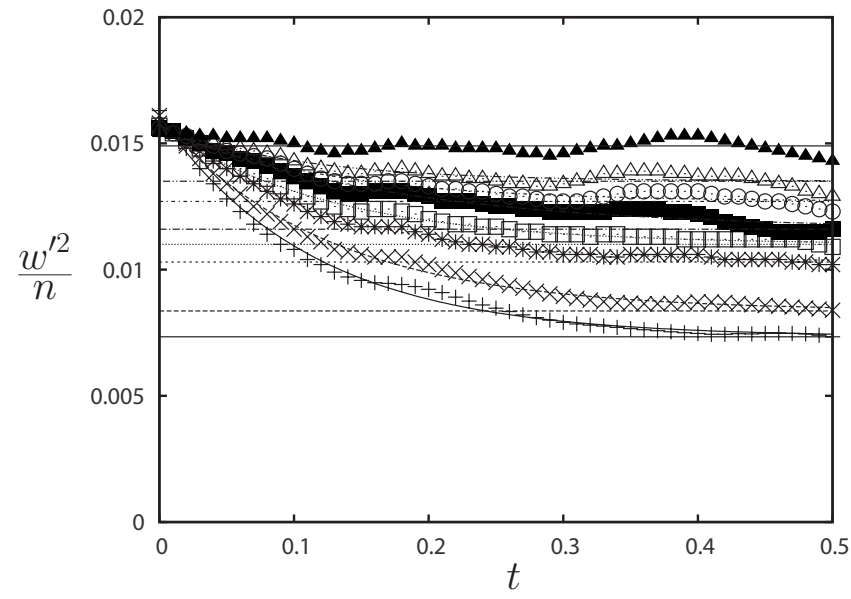

FIG. 6. The decay in time of velocity fluctuations in different stratifications; $b=0.04(+), 0.03(\times), 0.02\left(^{*}\right), 0.015(\square), 0.01(\square), 0.0075(\bigcirc), 0.005$ $(\triangle)$, and $0(\mathbf{\Delta})$; with number density $n=10^{3}$, number of Fourier modes in a horizontal direction $n_{F}=5$ and averaging over 40 realizations.

monitoring window during the simulations. The window is also well away from the sediment collecting at the bottom.

Figure 6 shows the decay in time of the velocity fluctuations in different stratifications. Note that we plot the mean square of the velocity fluctuations for the simulations and the root mean square for the experiments. Stratification $b$ varies from 0 to 0.04 , the latter giving a $40 \%$ variation in the concentration from bottom to top of the simulation box $(h=10)$. Each plot is the average over 40 realizations. Each simulation was made with $10^{4}$ particles, i.e., with a number density of $n=10^{3}$. Only five Fourier modes, $n_{F}=5$, were used in each horizontal direction, meaning that the smallest length scale of the flow resolved was 0.2 , twice the mean separation of the particles. As the velocities were inhibited at most by a factor of 2, the smallest length scale that needs be resolved in this first series of simulations was however 0.25, see Sec. I.

The velocity fluctuations all start at the nondimensional Poisson value of $w_{0}^{\prime 2}=0.015 n$, which corresponds to a dimensional value

$$
w_{0}^{\prime}=1.1 V_{S} \sqrt{\phi L / a},
$$

which we reported earlier. ${ }^{10}$ The numerical coefficient 0.015 depends on the stress-free boundary conditions used in the simulations. For nonslip boundaries, Mucha et al. ${ }^{15}$ found a coefficient of 0.48 . The velocity fluctuations then decay in time to a plateau, whose value decreases as the stratification is increased. Thus the stratification has no effect on the initial value of the velocity fluctuations, but does inhibit the long time value. In order to find the long time plateau values, each set of the numerical data is fitted to a simple exponential decay curve

$$
w_{\infty}^{\prime 2}+\left(w_{0}^{\prime 2}-w_{\infty}^{\prime 2}\right) \exp (-t / \tau) .
$$

This best fit is also plotted in Fig. 6, along with the long time value $w_{\infty}^{\prime 2}$. We shall be interested in these long time values and also the decay time scale $\tau$. For some values of the number density $n$ and the stratification $b$, the long time value $w_{\infty}^{\prime 2}$ was only achieved at times beyond the end of the simulations. The decaying exponential fit, however, allowed a 


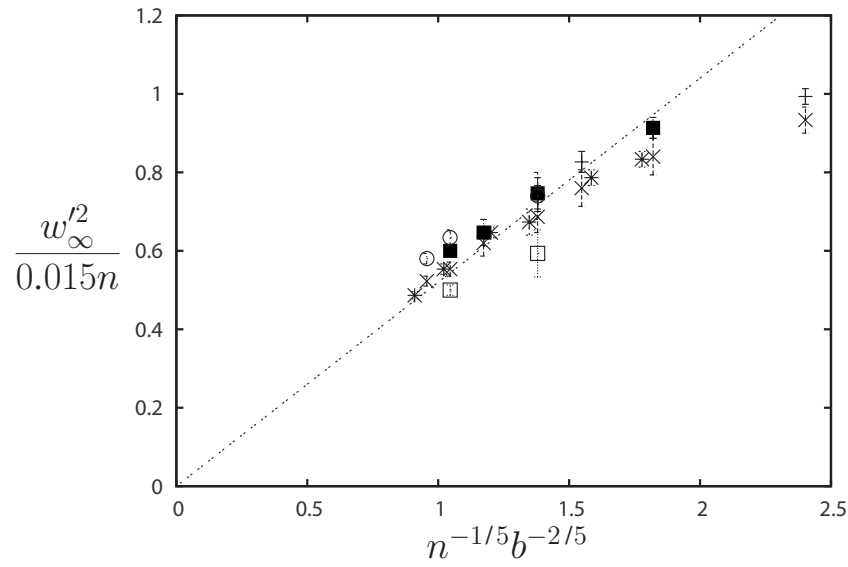

FIG. 7. The long time plateau values of the velocity fluctuations, $w_{\infty}^{\prime 2}$, divided by the initial Poisson value, $0.015 n$, as a function of stratification in the theoretical prediction $n^{-1 / 5} b^{-2 / 5}$; with Fourier modes $n_{F}=5$ and number density $n=125(+), 500(\times), 1000(*), 2000(\square) ; n_{F}=7$ and $n=500(\square)$; and $n_{F}=10$ and $n=500(\bigcirc)$. The line is $w^{\prime 2} / 0.015=0.52 n^{-1 / 5} b^{-2 / 5}$.

reasonable estimate to be found for its value. For each pair of numerical parameters, $n$ and $b$, eight independent runs were made, from which errors bars could be placed on the reported mean values of $w_{\infty}^{\prime 2}$ and $\tau$. At very low stratifications, a few of the runs could not be fitted sensibly to an exponential decay, resulting in larger error bars.

Figure 7 shows the long time plateau values of the velocity fluctuations $w_{\infty}^{\prime 2}$ normalized by the initial Poisson value $0.015 n$ as a function of the stratification $b$ and number density $n$ in the theoretical combination,

$$
n^{-1 / 5} b^{-2 / 5}=\left(\frac{4 \pi}{3}\right)^{1 / 5}\left(\frac{\beta_{c}}{\beta}\right)^{2 / 5}
$$

see Eqs. (4) and (3). It is seen that different Fourier resolutions give the same results within the error bars. It is seen that different values of the number density $n$ and stratification $b$ collapse onto the same curve when plotted as a function of $n^{-1 / 5} b^{-2 / 5}$. When the stratification is weak, $n^{-1 / 5} b^{-2 / 5}>2$, it has little effect on the velocity fluctuations. For stronger stratification, there is a linear dependence on the velocity fluctuations

$$
w_{\infty}^{\prime 2} / 0.015 n=0.52 n^{-1 / 5} b^{-2 / 5},
$$

which in dimensional terms is

$$
w_{\infty}^{\prime 2}=0.94 V_{\text {Poisson }}\left(\beta_{c} / \beta\right)^{1 / 5} .
$$

The relaxation time scale $\tau$ at which the velocity fluctuations decay from their initial homogeneous-suspension values to the stratified-inhibited values are plotted in Fig. 8. It is seen that different Fourier resolutions give the same results within the error bars. It is seen that the decay time is independent of the stratification $b$, so

$$
\tau \approx 4.3 / \sqrt{n},
$$

which in dimensional terms is

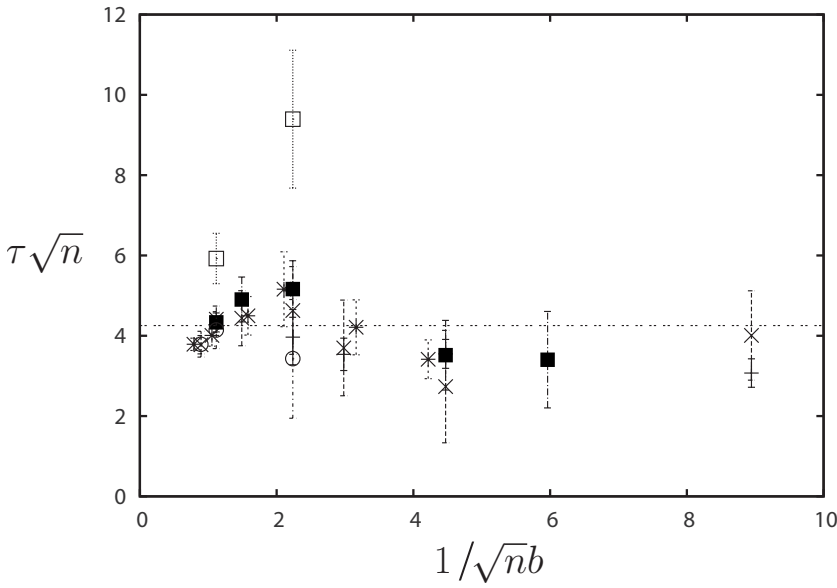

FIG. 8. The decay time scale $\tau$ as a function of the stratification $b$; with Fourier modes $n_{F}=5$ and number density $n=125(+), 500(\times), 1000(*)$, $2000(\square) ; n_{F}=7$ and $n=500(\square)$; and $n_{F}=10$ and $n=500(\bigcirc)$. The line is at 4.3 .

$$
\tau \approx 0.46 t_{S} \sqrt{L / a \phi}
$$

see Eq. (2). This decay time corresponds to a blob of size of the container falling half its height with its initial Poisson fluctuating velocity. One might have expected that the blob needs only fall to its neutral-buoyancy level $\sqrt{n} /(-\partial n / \partial z)=1 / \sqrt{n} b$, which would give $\tau \propto 1 / n b$. Such a decay rate would correspond to a linear line in the plot of Fig. 8. This is not seen.

\section{NUMERICAL RESULTS IN A MOVING FRONT}

The second series of numerical simulations start with a uniform suspension. As the sedimentation proceeds, a diffuse front descends separating the suspension below from clear fluid above. We study the inhibition of the velocity fluctuations within the front where the concentration varies vertically, i.e., the suspension is stratified. The passage of the descending front is viewed in a series of fixed vertical windows. Within each window, the velocity fluctuations are monitored in time, along with the mean concentration and its gradient by a best fit linear to the concentration.

In the second series of simulations, the number of Fourier modes was set equal to the number of particles $n_{F}^{3} h=N$. We found previously ${ }^{18}$ that at least this spatial resolution was required for the growth of the front to be independent of the resolution. (Mucha et al. ${ }^{15}$ may have had insufficient number of Fourier modes to resolve correctly the growth of the front.) The numerical simulations were made in a box of height $h=8$ with a mean sedimentation velocity $V=2$ for a time $0 \leq t \leq 3.5$. During the simulation, the middle of the front descends from $z=8$ to $z=1$ and the width of the diffuse front, defined by its first and third quartiles, increases to nearly 3. Observations in the window $0.5<z<1.5$ were therefore dominated by a second front rising from the sediment, and so this window was not used. Equally observations from a window $6.5<z<7.5$ were not used because at early times when the front was in this window, it was too narrow 


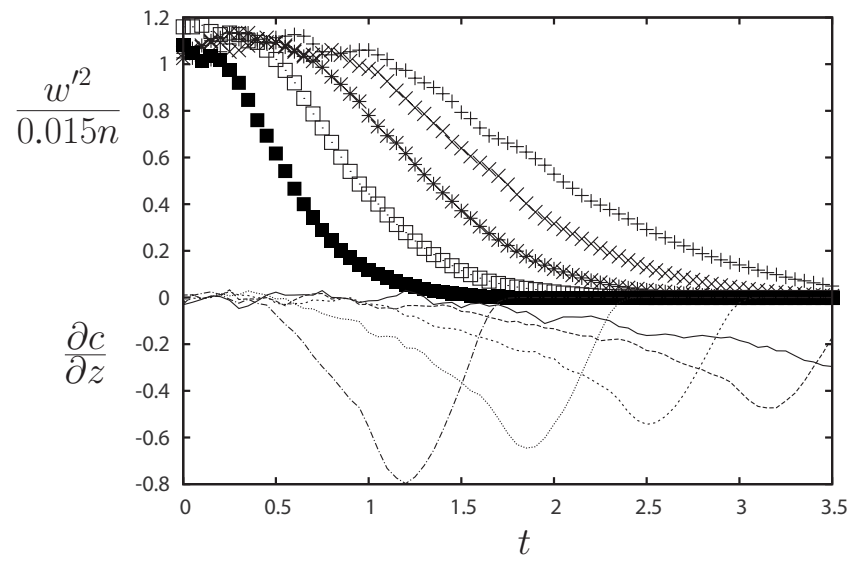

FIG. 9. Effect of the passing front on velocity fluctuations. Plotted as a function of time is the velocity fluctuations dividing the initial Poisson value at the top part, and at the bottom part the concentration gradient $\partial c / \partial z$. The windows are $[1.5,2.5](+),[2.5,3.5](\times),[3.5,4.5](*),[4.5,5.5](\square)$, and $[5.5,6.5](\mathbf{\square})$. The number density is $n=125$, the number Fourier modes in a horizontal is a direction $n_{F}=5$, the height $h=8$, and averaging over 320 realizations

for a linear fit across the whole window to represent well the sharp gradient. The descending front was therefore monitored in windows $[1.5,2.5]-[5.5,6.5]$.

Figure 9 shows the variation in time of the velocity fluctuations and the concentration gradient in the series of vertical windows, for a small simulation of 1000 particles but averaged over 320 realizations in order to produce moderately smooth plots. The successive arrival of the front in the different windows is seen in the lower part of the figure as the magnitude of the concentration gradient increases to a maximum and then decreases. The velocity fluctuations remain equal to their initial Poisson value of $w^{\prime 2} \approx 0.015 n$ until the front nears the corresponding window. The velocity fluctuations then begin to decrease.

The results in Fig. 9 are for a small system of only 1000 particles, corresponding to Fourier modes $n_{F}=5$. Larger simulations were made with $n_{F}=7,10$, and 12 , with 160,80 , and 40 realizations, respectively. The two largest were run only to time $t=1.5$ because the diffusive front grows faster, proportional to $n_{F}$ (when the number of particles is equal to the number of Fourier modes). Breaking the total number of realizations into eight independent runs allows error bars to be estimated in the calculated averaged quantities.

To test if the value of the local stratification in the descending front determines the value of the local velocity fluctuations, we plot in Fig. 10 the instantaneous values in each vertical window against one another. Before the front arrives, the concentration gradient in a window fluctuates around zero, taking negative and positive (unstably stratified) values in different realizations. This results in the rms error in the estimate of the concentration gradient exceeding its mean value. We omit these data with large errors, and wait until the front arrives and the rms error drops below $70 \%$ of the mean. The points on the right of Fig. 10 do have errors just below this level. A second problem occurs when the front is in a window: The best linear fit may not be a good approximation to the form of the vertical variation of the

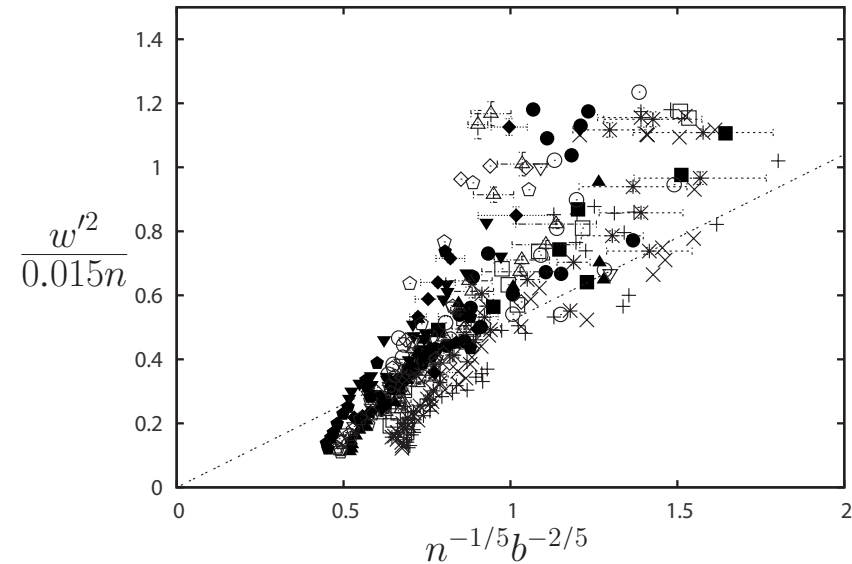

FIG. 10. The velocity fluctuations in the descending front divided by the initial Poisson value $w^{\prime 2} / 0.015 n$ as a function of the local stratification in the theoretical prediction $n^{-1 / 5} b^{-2 / 5}$. The windows are $[1.5,2.5](+\bigcirc \nabla)$, $[2.5,3.5](\times \bigcirc \diamond),[3.5,4.5][* \triangle \diamond]$ with error bars, $[4.5,5.5](\bullet \mathbf{\Delta} \bigcirc)$, and $[5.5,6.5](\square \nabla \bigcirc)$, with the three symbols corresponding to number densities $n=343,1000$, and 1728 . The line has slope 0.52 .

concentration in the window. This problem arises particularly on the top window when the front is sharp but also occurs when the front is at the point of exiting a window and much of the window contains no particles. To avoid such misleading estimates of the local concentration gradient, we omit data where the linear gradient would give more than $30 \%$ change from the mean concentration across the window, and also omit cases where the mean concentration is less than $1 \%$ of the initial value.

Figure 10 shows, with the data selected as described in the last paragraph, that the inhibition of the velocity fluctuations is determined by the stratification in the descending front. Data from the different windows, which have different variations in time, fall onto the same curve. Data from simulations with different number densities, or equivalently different spatial resolutions, fall onto the same curve. The different number densities have the front spreading at different rates. There is considerable scatter, with points with large horizontal error bars, at the top right, where the velocity fluctuations are not inhibited and the stratification is small. There might also be a small time delay before the velocities adapt to the increasing local stratification. On the other hand, the points at the bottom left have large concentration gradients, which may be underestimated by the best linear fit. If the gradients were larger than that estimated, the points would move to the left. With these reservations about the data at the extreme ends, the data fall reasonably near the simple relations found in the first series of simulations.

\section{CONCLUSIONS}

Both in our experiments and simulations we found that the initial velocity fluctuations follow the Poisson scaling (1),

$$
w_{0}^{\prime}=k V_{S} \sqrt{\phi L / a},
$$

and this is not affected by any initial stratification. The value of the coefficient was $k \approx 1.0$ in experiment ( 7$)$ and $k=1.1$ in 
simulation (9). The latter value is the theoretical value for tall boxes with a square section with impermeable no-stress boundaries. ${ }^{10}$ For no-slip boundaries, Mucha et al. ${ }^{15}$ gave the theoretical value 0.69 for tall boxes with square sections. In unstratified experiments in a $2 \times 3 \times 30$ cell, Tee et al. ${ }^{16}$ found 0.39 .

The initial velocity fluctuations were found to decay on a time scale corresponding to moving across the cross section with the Poisson velocity (2),

$$
\tau_{\text {relax }}=k t_{S} \sqrt{L / a \phi},
$$

whereas one might have anticipated an effect of stratification, e.g., Eq. (5). The value of the coefficient was $k \approx 0.3$ in experiment (8) and $k=0.46$ in simulation (11).

In the simulations, the initial fluctuations decay to a plateau value which depends on the large imposed stratification. The picture in the experiments is more complex. In the case of particles of batch $\mathrm{C}$ in the $4 \times 4 \mathrm{~cm}^{2}$, there is a strong gradient in the measuring window, see Fig. 1(d), which controls the velocity fluctuations in the plateau, see Fig. 3 (ם). In other cases where the stratification in the measuring window can be negative, see Fig. 1(b), or can alternate through the cell height, see Fig. 1(a), the plateau value is not controlled by the local stratification even though it is much larger than critical stratification, see other filled symbols in Fig. 3.

On the other hand, in the experiments the stratification controlled the fluctuations in the descending front. In the simulations, the same control occurred in the descending front, and also in the plateau after the early decay in those simulations with an imposed large initial stratification, as mentioned above. The velocity fluctuations were found to be affected by the stratification following the scaling ${ }^{11,13-16}$

$$
w^{\prime}=k V_{\text {Poisson }}\left(\beta_{c} / \beta\right)^{1 / 5} \text {. }
$$

The value of the coefficient was $k=0.8$ in experiment (6) and $k=0.93$ in simulation (10). In simulations, Mucha et al. ${ }^{15}$ found $k=0.87$ in a log-log plot (their Fig. 7) of their rather scattered data, while Tee et al. ${ }^{16}$ with less scatter found $k=0.39$ (their Fig. 4).

The crossover between the Poisson and stratified formulas occurs at

$$
\beta=k \beta_{c},
$$

with $\beta_{c}$ given by Eq. (3). The value of the coefficient was $k=0.43$ in our simulations and would be $k=0.33$ in our experiments, although we have no observation in the crossover region. Mucha and Brenner ${ }^{14}$ found $k=\frac{1}{2}$ for their simulations in a $\frac{1}{8} \times 1 \times 1$ box with no-slip boundaries. In some experiments in a $2 \times 3 \times 30$ cell with initial stratification, Tee et $a l .{ }^{16}$ found $k=\frac{1}{32}$. However unmeasured vertical variations in the concentration of sucrose may have dominated the measured density variations due the concentration of the particles.

In summary, the initial velocity fluctuations take the Poisson value, although we cannot be sure that mixing in the experiment produces a truly random initial state as in the simulations. The early decay, during which the density fluctuations fall a distance equal to the width of the section, is not affected by stratification. Stratification does inhibit the velocity fluctuations when the concentration gradient is quasiconstant over an extent greater than the measuring window, as occurs when the front is well developed. When the front is very sharp and in experiments when the concentration gradient fluctuates about zero through the height of the cell, the local value of the stratification does not control the velocity fluctuations even if greater than the critical value. In such circumstances, the global variation of the concentration throughout the container may be important. Equally, details of the initial state produced by the mixing may be relevant. ${ }^{19}$

\section{ACKNOWLEDGMENTS}

\section{D.C.G.}

Support from CONACyT is gratefully acknowledged by

${ }^{1}$ R. H. Davis and A. Acrivos, "Sedimentation of noncolloidal particles at low Reynolds numbers," Annu. Rev. Fluid Mech. 17, 91 (1985).

${ }^{2}$ G. K. Batchelor, "Sedimentation in a dilute dispersion of spheres," J. Fluid Mech. 52, 245 (1972)

${ }^{3}$ R. E. Caflisch and J. H. C. Luke, "Variance in the sedimenting speed of a suspension," Phys. Fluids 28, 759 (1985).

${ }^{4}$ E. J. Hinch, "Sedimentation of small particles," in Disorder and Mixing, edited by E. Guyon, J.-P. Nadal, and Y. Pomeau (Kluwer Academic, Dordrecht, 1988), p. 153.

${ }^{5}$ J. M. Ham and G. M. Homsy, "Hindered settling and hydrodynamic dispersion in quiescent sedimenting suspension," Int. J. Multiphase Flow 14, 533 (1988).

${ }^{6}$ H. Nicolai, B. Herzhaft, E. J. Hinch, L. Oger, and E. Guazzelli, "Particle velocity fluctuations and hydrodynamic self-diffusion of sedimenting nonBrownian spheres," Phys. Fluids 7, 12 (1995).

${ }^{7}$ H. Nicolai and E. Guazzelli, "Effect of the vessel size on the hydrodynamic diffusion of sedimenting spheres," Phys. Fluids 7, 3 (1995).

${ }^{8}$ P. N. Segrè, E. Herbolzheimer, and P. M. Chaikin, "Long-range correlations in sedimentation," Phys. Rev. Lett. 79, 2574 (1997).

${ }^{9}$ É. Guazzelli, "Evolution of particle-velocity correlations in sedimentation," Phys. Fluids 13, 1537 (2001).

${ }^{10}$ L. Bergougnoux, S. Ghicini, É. Guazzelli, and E. J. Hinch, "Spreading fronts and fluctuations in sedimentation," Phys. Fluids 15, 1875 (2003).

${ }^{11}$ J. Bławdziewicz, private communication (ca. 1995).

${ }^{12}$ J. H. C. Luke, "Decay of velocity fluctuations in a stably stratified suspension," Phys. Fluids 12, 1619 (2000).

${ }^{13}$ S.-Y. Tee, P. J. Mucha, L. Cipelletti, S. Manley, M. P. Brenner, P. N. Segrè, and D. A. Weitz, "Nonuniversal velocity fluctuations of sedimenting particles," Phys. Rev. Lett. 89, 054501 (2002).

${ }^{14}$ P. J. Mucha and M. P. Brenner, "Diffusivities and front propagation in sedimentation," Phys. Fluids 15, 1305 (2003).

${ }^{15}$ P. J. Mucha, S.-Y. Tee, D. A. Weitz, B. I. Shraiman, and M. P. Brenner, "A model for velocity fluctuations in sedimentation," J. Fluid Mech. 501, 71 (2004).

${ }^{16}$ S.-Y. Tee, P. J. Mucha, M. P. Brenner, and D. A. Weitz, "Velocity fluctuations of initially stratified sedimenting spheres," Phys. Fluids 19, 113304 (2007).

${ }^{17}$ D. Chehata Gómez, L. Bergougnoux, E. J. Hinch, and É. Guazzelli, “On stratification control of the velocity fluctuations in sedimentation," Phys. Fluids 19, 098102 (2007).

${ }^{18}$ D. Chehata Gómez, L. Bergougnoux, É. Guazzelli, and E. J. Hinch, "Spreading fronts in sedimentation of dilute suspension of spheres," Phys. Fluids 20, 023302 (2008).

${ }^{19}$ L. Bergougnoux and É. Guazzelli, "Non-Poisson statistics of settling spheres," Phys. Fluids 21, 091701 (2009). 